\title{
La credibilidad de los medios y de la información sobre la salud entre los habitantes de la Ciudad de México durante la pandemia COVID-19 (julio-diciembre 2020)
}

\section{The credibility of media and health information among the inhabitants of Mexico City during the COVID-19 pandemic (July-December 2020)}

Sergio Adrián Escalona Villegas*

Investigador independiente

Orleans núm. 16, Col. Lomas Estrella, Iztapalapa,

C.P. 09890, Ciudad de México, México

Raúl Santos Morales**

Universidad Anáhuac México

Av. Universidad Anáhuac núm. 46, Colonia Lomas Anáhuac,

C. P. 52786, Huixquilucan, Estado de México, México

\section{Editor: Rogelio del Prado Flores}

Fecha de recepción: 16 de marzo de 2021

Fecha de aceptación: 12 de mayo de 2021 sergioescalona@saev12.com

https://orcid.org/0000-0002-5012-3418

raul.santos@anahuac.mx https://orcid.org/0000-0002-6128-9616

\section{RESUMEN}

En medio de un panorama mediático amplio y diverso, entre los viejos y los nuevos medios, no está muy clara la manera en que las personas se informan sobre temas de la salud y mucho menos se conoce la credibilidad que se atribuye hacia los medios y hacia sus contenidos, por lo que se realizó una investigación cuantitativa de corte exploratorio y descriptivo a 15I habitantes de la Ciudad de México mediante la aplicación de un cues-

* Licenciado en Diseño Publicitario, especialista en Creatividad y Estrategia Publicitaria y en Multimedia por la Escuela de Diseño del Instituto Nacional de Bellas Artes (EDINBA), Maestro en Diseño Creativo Digital por el Centro Universitario de Comunicación (CUC). Tiene experiencia como profesor de licenciatura impartiendo la clase de Mercadotecnia para nuevos medios en el SAE Institute y profesor de maestría en la Universidad Anáhuac del Sur. Actualmente es estudiante de Doctorado en Investigación de la Comunicación de la Universidad Anáhuac México y director creativo del despacho de diseño SAEVI2 Servicios Creativos, del cual también es fundador, y ha desarrollado comunicación en temas de salud visual por más de una década para el sector privado.

** Licenciado en Publicidad, Maestro en Diseño Gráfico y Doctor en Comunicación Aplicada. De 1997 a 2006 trabajó en diversas agencias de publicidad para más de 2000 marcas nacionales y transnacionales. Actualmente es profesor-investigador del Centro de Investigación para la Comunicación Aplicada (CICA) de la Facultad de Comunicación dentro de la Universidad Anáhuac México. 
tionario durante la pandemia provocada por la COVID-I9 (julio-diciembre 2020), encontrándose que las páginas web especializadas son la principal fuente de información y las que poseen una mayor credibilidad en temas de salud; sin embargo, con una muestra tan pequeña, los resultados no son concluyentes y es necesario realizar una investigación más amplia, incluso de corte cualitativo para profundizar más en los elementos que conducen a las personas a buscar y evaluar este tipo de información.

Palabras clave: credibilidad, información de la salud, medios, nuevos medios, pandemia.

\section{ABSTRACT}

In the midst of a wide and diverse media landscape, between old, new, and new new media, it is not very clear how people find out about health information, much less the credibility that exists towards the media and towards its contents, for which a quantitative explor-

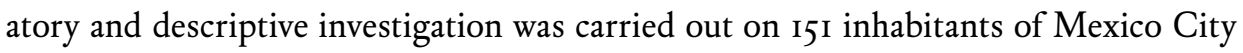
by applying a questionnaire during the pandemic caused by CoviD-I9 (July-December 2020), finding that the specialized web pages are the main source of information and those with greater credibility in health matters, however, given such a small sample, the results are not conclusive and it is necessary to carry out a broader investigation, even qualitative in order to deepen more on the elements that lead people to seek and evaluate this type of information.

Key words: credibility, health information, media, new media, pandemic.

\section{INTRODUCCIÓN}

Durante 2020, la pandemia provocada por la COVID-I9 situó a la salud como el tema más importante en todo el mundo, introdujo nuevas ideas y creencias, e hizo circular abundante información y comunicación sobre lo saludable y sobre este nuevo virus que, en México, hasta el 30 de diciembre de 2020, había provocado i 413935 casos de personas contagiadas y 124897 defunciones a nivel nacional (Secretaría de Salud, 2020).

Los principales signos de la COVID-I9, como la tos, fiebre, dolor de cabeza, dolor o ardor de garganta, ojos rojos, dolores en músculos o articulaciones (malestar general) y/o problemas para respirar o falta de aire en los pulmones (Secretaría de Salud, 202I) provocan daños en los pulmones, corazón, riñones, hígado, sistema nervioso, cerebro, piel y tracto 
gastrointestinal, por lo que la COVID-I9 puede ser catalogada como una enfermedad multisistémica con una afectación principal en el sistema respiratorio (BBC News, 2020; Fox, 2020), síntomas y efectos que desde el inicio de la pandemia en México, a finales de febrero de 2020 , se fueron dando a conocer a través de diferentes canales y medios de información análogos y digitales (MSP, 2020).

Sin embargo, con la aparición de servicios médicos a través de internet, mediante el apoyo de páginas web, redes sociodigitales y aplicaciones digitales (Hawn, 2009), el cambio tan abrupto del panorama de los medios, la proliferación de recursos sobre salud y el fácil acceso a los medios y a diferentes plataformas a través de dispositivos móviles, la difusión de información sobre salud se ha vuelto una tarea mucho más compleja (Clayman, Manganello, Viswanath, Hesse \& Arora, 20I0).

El panorama mediático se ha vuelto muy amplio desde finales del siglo Xx. Los viejos medios, como la prensa escrita -periódicos y revistas-, la radio y la televisión, sólo permitían recibir y consumir información con la posibilidad de socializarla de manera individual en el mundo real. En cambio, los nuevos medios, que surgen con la masificación de Internet desde finales del siglo xx, hicieron posible recibir, consumir, indagar, evaluar, publicar y propagar información a través de diversos espacios y herramientas, como páginas web, foros de discusión, blogs, microblogs, buscadores y correos electrónicos, principalmente, que permitían socializar individualmente la información en el mundo real y en el mundo virtual con bastante amplitud. Pero ahora, con los nuevos nuevos medios, es posible no sólo recibir, consumir, indagar, evaluar, publicar y propagar información, sino también crear, producir, criticar, etiquetar y comentar información a través de diversos canales y plataformas, que permiten niveles muy altos y casi ilimitados de socialización individual de la información en el mundo real, pero principalmente en el mundo virtual, a través de Facebook, Twitter, Instagram, Pinterest, etc. Por lo tanto, el panorama mediático ahora puede ser entendido a partir de los viejos medios, los nuevos medios y los nuevos nuevos medios, a pesar de que se traslapan entre sí y cada vez se vuelve más difícil reconocer los límites entre ellos (Levinson, 2013).

Los viejos medios aún son importantes en nuestro país, ya que $95 \%$ de los mexicanos cuenta con televisión, $48 \%$ con radio, estéreo o grabadora, y $34 \%$ con teléfono fijo. Los nuevos medios van creciendo poco a poco: $32 \%$ de los mexicanos cuenta con computadora de escritorio o computadora portátil y el $20 \%$ con Blu-ray o DVD, pero el crecimiento se está dado en los nuevos nuevos medios: $86 \%$ de los mexicanos dispone de un teléfono móvil, $19 \%$ de una tableta digital, $16 \%$ de otros dispositivos inteligentes para conectarse a internet, $15 \%$ de una consola de videojuegos y el $1 \%$ de dispositivos de realidad virtual. De esta manera, tanto los nuevos nuevos medios como los contenidos audiovisuales se han 
vuelto cada vez más relevantes en los últimos años dentro de la población mexicana (IFT, 20I9), algo que es notorio en la forma de ver televisión en México, ya que la televisión abierta tiene 69.3 millones de televidentes a nivel nacional, mientras que la televisión de paga cuenta con 56.7 millones de televidentes a nivel nacional (IFT, 20I9) y YouTube, la principal plataforma para ver videos en México, tiene 120 millones de usuarios a nivel nacional (Kemp, 202I).

En 2009, el 30\% de los habitantes del país eran usuarios de internet (IAB, 20I9); en 2020 , con una población de 126 OI4 O24 habitantes (INEGI, 2O2I), el porcentaje asciende a un $69 \%$ de la población nacional, es decir, 84.I millones de mexicanos son usuarios de internet. Facebook siempre ha sido líder en nuestro país como espacio de interacción sociodigital, alcanzado al 94\% de los usuarios de internet a nivel nacional (79 millones de mexicanos), por encima de WhatsApp (89\% de usuarios, 74 millones de mexicanos), Instagram (71\% de usuarios, 59 millones de mexicanos), Twitter (6I\% de usuarios, $5 \mathrm{I}$ millones de mexicanos), Pinterest (46\% de usuarios, 38 millones de mexicanos), LinkedIn (36\% de usuarios, 30 millones de mexicanos), Snapchat ( $35 \%$ de usuarios, 29 millones de mexicanos) o Skype (32\% de usuarios, 26 millones de mexicanos), aunque un espacio audiovisual como YouTube cuenta con el $96 \%$ de usuarios del país, es decir, 80 millones de mexicanos (Kemp, 202I).

Actualmente, 7 de cada Io mexicanos usa internet, tiene en promedio 5 redes sociodigitales, utiliza de 4 a 5 dispositivos para conectarse a internet, siendo el principal dispositivo el teléfono móvil (90\% de los usuarios), seguido de la computadora portátil o de escritorio ( $75 \%$ de usuarios), las pantallas inteligentes ( $72 \%$ de los usuarios), las tabletas digitales $(53 \%$ de los usuarios) y las consolas de videojuegos ( $53 \%$ de los usuarios), dispositivos que utilizan para entrar a redes sociodigitales ( $90 \%$ de los usuarios), revisar y enviar correos electrónicos ( $84 \%$ de los usuarios), buscar información ( $76 \%$ de los usuarios), chatear o mandar mensajes instantáneos (61\% de los usuarios), escuchar o descargar música (56\% de los usuarios) y utilizar servicios bancarios (47\% de los usuarios) (IAB, 2O2O).

En México buscar información sobre esta pandemia fue algo importante en un inicio, ya que la palabra "Coronavirus" fue la tendencia principal de las búsquedas de internet durante 2020, obtuvo su máximo nivel de búsqueda a principios de abril de 2020, disminuyó a principios de mayo y desapareció casi por completo a mediados de septiembre (Google, 202I), mientras que la pregunta más buscada en México a través de internet durante 2020 fue “¿Qué es el coronavirus?”, que alcanzó su nivel más alto de búsqueda a mediados de enero de 2020 , disminuyó a principios de diciembre y desapareció casi por completo a finales de ese mismo mes (Google, 202I). 


\section{ESTADO DEL ARTE}

La información sobre la salud depende de las fuentes, espacios y canales de información, e incluso de la credibilidad y confianza en las instituciones gubernamentales o sanitarias (Cheung \& Tse, 2008; van der Weerd, Timmermans, Beaujean, Oudhoff \& van Steenbergen, 20II; Palanisamy, Gopichandran \& Kosalram, 20I8), credibilidad que ha disminuido con el paso del tiempo (Jackson, Peterson, Blake, Coa \& Chou, 20I9) y ha quedado en manos de los medios de información (Clayman, Manganello, Viswanath, Hesse \& Arora, 20Io), aunque cada vez la información de Internet se ha vuelto una fuente más creíble (Vidgen, Sims \& Powell, 20I3), ya que su valor no sólo radica en ser abundante y estar disponible en cualquier momento, sino en el apoyo emocional y social que se obtiene de otras personas dentro de una página, foro, comunidad o red sociodigital en internet (Hajli, Sims, Featherman \& Love, 2015) a pesar de que no existe ningún control ni verificación sobre la información que se publica en internet y, por lo tanto, también existe mucha desinformación (Shah et al., 20I9; Chou, Gaysynsky \& Cappella, 2020).

Los estudios sobre credibilidad surgen en Estados Unidos durante la década de los 40, en el campo de la investigación sobre los medios masivos de comunicación, con el trabajo pionero de Carl Hovland, Irving Janis y Harold Kelley. El interés por este tema surgió durante esa época con el fin de aumentar el apoyo de las personas hacia la guerra, estudiar el cambio de actitudes y desarrollar una teoría de la persuasión (Self, 1996). A partir de entonces, se han originado múltiples investigaciones, que en los últimos años se han vuelto mucho más específicas, puesto que se enfocan en la credibilidad de los mensajes de los nuevos medios y de los nuevos nuevos nuevos medios, así como en la credibilidad de los medios como fuentes de información (Sbaffi \& Rowley, 20I7; Shin, Lee \& Hwang, 20I7; Thon \& Jucks, 20I7; Borah \&Xiao, 2018; De Meulenaer, De Pelsmacker \&Dens, 2018; Embacher, McGloin \& Richards, 20I8; Klawitter \& Hargittai, 20I8; Machackova \& Smahel, 20I8; Popescu et al., 20I8; Valizadeh-Haghi, Rahmatizadeh, Ansari, \& Hamzehei, 20I8; Choi, 2020; Masílamani, Sriram \& Rozario, 2020; Song, Zhang \& Yu, 2020; Chang, Zhang \& Gwizdka, 202I), investigaciones que recalcan la vinculación entre la credibilidad y las percepciones, la utilidad percibida, la construcción de creencias y comportamientos, la experiencia de la fuente del mensaje, la facilidad para obtener la información, la calidad de la información, la experiencia en el uso de tecnologías digitales, los procesos de búsqueda de información, la retroalimentación recibida, los motivos personales, el diseño de la información, además de las características biológicas de los autores, permiten la construcción de una identidad digital de los emisores y los usuarios.

Más recientemente, estos estudios se han ido expandiendo hacia la credibilidad de las organizaciones e instituciones de salud (Ihlen, 2020; Xiang, Guo \& Liu, 2020), señalando 
que la credibilidad implica estrategia, negociación, un efecto moderador, e incluso la moral pública de cada país o sociedad. En general, todos estos estudios apuntan a una realidad muy compleja donde la credibilidad depende de múltiples factores, pero especialmente de cada individuo y su contexto, aunque ninguna de estas investigaciones ha identificado cuáles son los principales medios que utilizan las personas para informarse sobre temas de salud, lo cual es el objetivo principal de esta investigación.

\section{MARCO TEÓRICO}

Se entiende por credibilidad de la fuente los juicios que tiene una persona sobre la aceptación de un mensaje y del emisor de dicho mensaje (Hovland, Janis, \& Kelley, I953), de tal manera que existen dos rutas básicas para generar esa credibilidad, aunque hoy se sabe que la credibilidad también depende de varios factores, como el tipo de fuente que se esté evaluando, el contexto de la evaluación, los canales de información, la atención de las personas hacia el mensaje, la comprensión del mensaje y otras múltiples variables (Cronkhite \& Liska, I976; Delia, I976; Petty \& Cacioppo, I981; Gunther, I988, I992; Stamm \& Dube, I994; Gass \& Seiter, 1999), por lo que, en algunos casos, el mensaje es más importante que la fuente de información o que el emisor del mensaje, sobre todo cuando hay una alta implicación en el tema y una alta relevancia personal, como pueden ser los temas sobre salud, lo que aumenta la motivación de las personas para examinar de manera más detallada el contenido de un mensaje (Petty \& Cacioppo, I981; Benoit, I987; O’Keefe, I990; Eagly \& Chaiken, 1993).

Por otro lado, la credibilidad de un medio específico depende principalmente de sus características tecnológicas y estructurales (Carter \& Greenberg, 1965; Gaziano \& McGrath, I986; Rimmer \& Weaver, I987), aunque hoy se sabe que las personas usan diferentes puntos de referencia para evaluar la credibilidad de varios medios al mismo tiempo, así como del nivel de información que ofrecen -información local, nacional o internacional-, y del tipo de temas que buscan (Greenberg \& Roloff, 1974; Abel \& Wirth, 1977; Lee, 1978; Reagan \& Zenalty, I979; Gantz, I98I; Gaziano \& McGrath, I986; Meyer, I988; Newhagen \& Nass, 1989). Sin embargo, un determinado medio puede tener mayor credibilidad cuando tiene información muy específica, lo cual también dependerá del atractivo y dinamismo del mismo medio (Flanagin \& Metzger; 2007), algo que puede marcar notables diferencias entre los viejos medios, los nuevos medios y los nuevos nuevos medios. 


\section{MÉTODO Y DESARROLLO}

Como parte del de un proyecto más amplio, de julio a diciembre de 2020, se realizó una investigación exploratoria de corte cuantitativo y descriptivo a I5 I personas $(n=15 \mathrm{I})$, lo que implicó un muestreo no probabilístico por conveniencia con las siguientes características: a) ser mayores de edad, y b) cohabitar con otras personas dentro de la Ciudad de México. A esta muestra se le aplicó un cuestionario que incluía tres preguntas de opción múltiple y dieciséis preguntas con una escala aditiva de evaluaciones sumarias o escala de Likert de cinco categorías, con el objetivo de descubrir cuáles eran los principales medios que utilizaban las personas para informarse sobre temas de salud, además de contrastar la credibilidad atribuida a cada medio, lo que ayudó a establecer las implicaciones sobre la información de la salud durante la pandemia provocada por la COVID-I9.

\section{RESULTADOS}

La muestra incluyó $54 \%$ de mujeres ( 82 personas) y $46 \%$ de hombres (69 personas) con una edad media de 32 años y un rango de 33 años, una edad mínima de i 8 años y una edad máxima de 5I años.

Se encontró una gran diversidad en cómo las personas obtienen información sobre temas de salud. De manera particular, el principal medio por el cual las personas buscan ese tipo de información es a través de páginas web especializadas en salud (I7.5\%, 26 personas), mientras que los medios menos utilizados son los posters/carteles, folletos y volantes ( $2 \%$, 3 personas).

De manera general, las personas utilizan los viejos medios (39\%, 6o personas) para informarse sobre temas de salud, seguidos de los nuevos nuevos medios ( $36 \%, 54$ personas) y de los nuevos medios ( $24 \%, 35$ personas), pero llama la atención que el ı\% (2 personas) no recurre a ningún medio, sino que buscan información a través de conocidos del área de la salud (Tabla I).

Respecto a la credibilidad de los medios sobre temas de salud, de manera general son los nuevos medios, como las páginas web especializadas, blogs especializados y correos electrónicos, los que tienen mayor credibilidad para las personas, seguidos de los viejos medios, como las revistas, los periódicos, los pósters/carteles, los volantes y los folletos, mientras que los nuevos nuevos medios como Facebook, Twitter, WhatsApp, Instagram, Pinterest o YouTube son los menos confiables para las personas. 
TABLA 1. MEDIOS UTILIZADOS PARA INFORMARSE SOBRE TEMAS DE SALUD

\begin{tabular}{|c|c|c|}
\hline \multicolumn{2}{|c|}{ MEDIOS } & PORCENTAJE/PERSONAS \\
\hline \multirow{7}{*}{$\begin{array}{l}\text { Viejos medios } \\
(39 \%, 60 \text { personas) }\end{array}$} & Televisión & $14 \%$ (21 personas) \\
\hline & Radio & 7\%(11 personas) \\
\hline & Periódicos & 7\% (11 personas) \\
\hline & Revistas & $5 \%$ (8 personas) \\
\hline & Pósters/carteles & $2 \%$ (3 personas) \\
\hline & Volantes & $2 \%$ (3 personas) \\
\hline & Folletos & $2 \%$ (3 personas) \\
\hline \multirow{3}{*}{$\begin{array}{l}\text { Nuevos medios } \\
(24 \%, 35 \text { personas })\end{array}$} & Páginas web especializadas & $17.5 \%$ (26 personas) \\
\hline & Blogs especializados & $3.5 \%$ (5 personas) \\
\hline & Correo electrónico & $3 \%$ (4 personas) \\
\hline \multirow{6}{*}{$\begin{array}{l}\text { Nuevos nuevos medios } \\
(36 \%, 54 \text { personas })\end{array}$} & Facebook & $15 \%$ (23 personas) \\
\hline & Instagram & $6 \%$ (9 personas) \\
\hline & Twitter & $5 \%$ (8 personas) \\
\hline & WhatsApp & $4 \%$ (6 personas) \\
\hline & Pinterest & $3 \%$ ( 4 personas) \\
\hline & YouTube & $3 \%$ ( 4 personas) \\
\hline \multirow[t]{2}{*}{ Otros (1\%, 2 personas) } & Conocidos del área de salud & $1 \%$ (2 personas) \\
\hline & Total $=16$ medios & Total $=100 \%$ (151 personas $)$ \\
\hline
\end{tabular}

FUENTE: ELABORACIÓN PROPIA.

De manera particular, los medios que tienen mayor credibilidad para las personas sobre temas de salud son las páginas web especializadas y los blogs especializados (nuevos me- 
dios) seguido de las revistas (viejos medios), mientras que los medios con menor credibilidad son Instagram, Pinterest y Twitter (nuevos nuevos medios) (Tabla 2).

TABLA 2. CREDIBILIDAD DE LOS MEDIOS UTILIZADOS PARA ENTERARSE SOBRE TEMAS DE SALUD

\begin{tabular}{|c|c|c|}
\hline TIPO DE MEDIOS & \multicolumn{2}{|c|}{ CREDIBILIDAD } \\
\hline VIEJOS MEDIOS & BAJA & ALTA \\
\hline Revistas & $\begin{array}{c}35 \% \\
\text { (53 personas) }\end{array}$ & $\begin{array}{c}65 \% \\
\text { (98 personas) }\end{array}$ \\
\hline Periódicos & $\begin{array}{c}85 \% \\
\text { (128 personas) }\end{array}$ & $\begin{array}{c}15 \% \\
\text { (23 personas) }\end{array}$ \\
\hline Folletos & $\begin{array}{c}46 \% \\
\text { (69 personas) }\end{array}$ & $\begin{array}{c}54 \% \\
\text { (82 personas) }\end{array}$ \\
\hline Volantes & $\begin{array}{c}48 \% \\
\text { (72 personas) }\end{array}$ & $\begin{array}{c}52 \% \\
\text { (79 personas) }\end{array}$ \\
\hline Pósters/carteles & $\begin{array}{c}51 \% \\
\text { (77 personas) }\end{array}$ & $\begin{array}{c}49 \% \\
\text { (74 personas) }\end{array}$ \\
\hline Televisión & $\begin{array}{c}55 \% \\
\text { (83 personas) }\end{array}$ & $\begin{array}{c}45 \% \\
\text { (68 personas) }\end{array}$ \\
\hline Radio & $\begin{array}{c}73 \% \\
\text { (110 personas) }\end{array}$ & $\begin{array}{c}27 \% \\
\text { (41 personas) }\end{array}$ \\
\hline NUEVOS MEDIOS & BAJA & ALTA \\
\hline Páginas web especializadas & $\begin{array}{c}20 \% \\
\text { (30 personas) }\end{array}$ & $\begin{array}{c}80 \% \\
\text { (121 personas) }\end{array}$ \\
\hline Blogs especializados & $\begin{array}{c}27 \% \\
\text { (41 personas) }\end{array}$ & $\begin{array}{c}73 \% \\
\text { (110 personas) }\end{array}$ \\
\hline Correo electrónico & $\begin{array}{c}90 \% \\
\text { (136 personas) }\end{array}$ & $\begin{array}{c}10 \% \\
\text { (15 personas) }\end{array}$ \\
\hline
\end{tabular}




\begin{tabular}{|c|c|c|}
\hline NUEVOS NUEVOS MEDIOS & BAJA & ALTA \\
\hline YouTube & $\begin{array}{c}52 \% \\
\text { (79 personas) }\end{array}$ & $\begin{array}{c}48 \% \\
\text { (72 personas) }\end{array}$ \\
\hline WhatsApp & $\begin{array}{c}74 \% \\
\text { (112 personas) }\end{array}$ & $\begin{array}{c}26 \% \\
\text { (39 personas) }\end{array}$ \\
\hline Facebook & $\begin{array}{c}81 \% \\
\text { (122 personas) }\end{array}$ & $\begin{array}{c}19 \% \\
\text { (29 personas) }\end{array}$ \\
\hline Twitter & $\begin{array}{c}95 \% \\
\text { (143 personas) }\end{array}$ & $\begin{array}{c}5 \% \\
\text { (8 personas) }\end{array}$ \\
\hline Instagram & $\begin{array}{c}96 \% \\
\text { (145 personas) }\end{array}$ & $\begin{array}{c}4 \% \\
\text { (6 personas) }\end{array}$ \\
\hline Pinterest & $\begin{array}{c}98 \% \\
\text { (148 personas) }\end{array}$ & $\begin{array}{c}2 \% \\
\text { (3 personas) }\end{array}$ \\
\hline
\end{tabular}

FUENTE: ELABORACIÓN PROPIA.

\section{ANÁLISIS Y DISCUSIÓN DE RESULTADOS}

Los resultados obtenidos muestran que existe una gran diversidad en la manera en que las personas se informan sobre temas de la salud, lo que les permite tener y evaluar diferentes puntos de referencia entre diferentes medios, pero la información sobre salud, al ser un tema específico y especializado, muestra un panorama en el que los nuevos nuevos medios, como Facebook, Twitter, WhatsApp, Instagram, Pinterest y YouTube, están ligeramente por debajo de los viejos medios y de los nuevos medios como fuente de consulta, y la credibilidad de los medios digitales se pone en entredicho, con excepción de las páginas web especializadas en salud, que fueron las únicas que tuvieron una gran credibilidad durante la pandemia provocada por la COVID-I9.

Una página web especializada en salud puede ofrecer temas muy particulares de manera inmediata, en cualquier día y a cualquier hora, sobre todo porque detrás de ella está un equipo de profesionales, expertos en diferentes campos y áreas de la salud, lo que ayuda a incrementar su credibilidad, y aunque no es tan dinámica como un video o una animación, se puede volver más atractiva con la amplia información que ofrece, por lo que las páginas web especializadas en salud, además de ser los principales medios y fuentes de información 
sobre salud entre una población joven de la Ciudad de México con un promedio de edad de 32 años, cuentan con la mayor credibilidad de todos los medios, a pesar de que Facebook y la televisión son otros medios importantes a través de los cuales ese sector de la población se informa sobre temas de salud.

\section{CONCLUSIONES}

La vasta oferta de información sobre salud ha generado un panorama mediático demasiado fragmentado, en el que la credibilidad del medio y de sus contenidos se vuelve relevante para las personas, y depende no sólo de las características tecnológicas y estructurales del medio sino también del atractivo que ofrezcan.

A diferencia de un video en YouTube, Instagram, Pinterest o Facebook o de un mensaje en Twitter o WhatsApp, las páginas web especializadas, al permitir datos duros, comparar datos mediante tablas y gráficas e incluir una amplia información, generan un mayor uso y una mayor credibilidad; sin embargo, con una muestra tan pequeña, los resultados obtenidos no son concluyentes, por lo que una investigación más amplia ayudará a aclarar mucho más el panorama mediático que existe detrás de la información sobre la salud, no sólo a través de una investigación cuantitativa, sino a través de una investigación cualitativa que permita descubrir los elementos que conducen a las personas a buscar y evaluar este tipo de información entre los viejos, nuevos y nuevos nuevos medios, no sólo durante la pandemia provocada por la COVID-I9 sino después de que haya sido superada, con la finalidad de contrastar si existe o no un cambio en las fuentes de información y en la credibilidad de las mismas.

\section{REFERENCIAS}

Abel, J. D. \& Wirth, M. O. (1977). Newspapers vs. TV credibility for local news. Fournalism Quarterly, 54(2), 37I-375. https://doi.org/I0.II77/I07769907705400223

BBC News (25 de mayo de 2020). Coronavirus | De enfermedad respiratoria a multisistémica: cómo en pocas semanas cambió radicalmente lo que sabemos sobre la COVID-I9. $B B C$ News. Mundo. Noticias. Recuperado de: https://bbc.in/3osoGXz

Benoit, W. L. (1987). Argumentation and credibility appeals in persuasion. Soutbern Speech Communication Fournal, 52(2), I8I-197. https://doi.org/I0.1080/I0417948709372687

Borah, P., \& Xiao, X. (2018). The importance of "likes": the interplay of message framing, source, and social endorsement on credibility perceptions of health information on Facebook. Fournal 
of Health Communication, 23(4), 399-4II. https://doi.org/10.1080/10810730.2018.1455770

Carter, R.F. \& Greenberg, B.S. (1965). Newspapers or television: Which do you believe? Fournalism Quarterly, 42(I), 29-34. https://doi.org/10.1177/107769906504200104

Chang, Y. S., Zhang, Y. \& Gwizdka, J. (202I). The effects of information source and eHealth literacy on consumer health information credibility evaluation behavior. Computers in Human Bebavior, II5, I-33. https://doi.org/I0.IOI6/j.chb.2020.106629

Cheung, C. \& Tse, J. (2008). Institutional trust as a determinant of anxiety during the SARS crisis in Hong Kong. Social Work in Public Health, 23(5), 4I-54. https://doi. org/10.1080/19371910802053224

Choi, W. (2020). Older adults' credibility assessment of online health information: an exploratory study using an extended typology of web credibility. Fournal of the Association for Information Science \& Technology (FASIST), 7I(II), I295-1307. https://doi.org/10.1002/asi.2434I

Chou, S. W. Y., Gaysynsky, A. \& Cappella, J. N. (2020). Where we go from here: health misinformation on social media. American Fournal of Public Health, IIO $\left(\mathrm{S}_{3}\right), \mathrm{S}_{273}-\mathrm{S}_{275}$. https://doi. org/10.2105/AJPH.2020.305905

Clayman, M. L., Manganello, J. A., Viswanath, K., Hesse, B. W. \& Arora, N. K. (2010). Providing health messages to Hispanics/Latinos: understanding the importance of language, trust in health information sources, and media use. Fournal of Health Communication, I5(sup3), 252-263. https://doi.org/10.1080/10810730.2010.522697

Cronkhite, G. \& Liska, J. (1976). A critique of factor analytic approaches to the study of credibility. Communication Monograpbs, 43(2), 91-107. https://doi.org/I0.1080/03637757609375920

Delia, J. G. (1976). A constructivist analysis of the concept of credibility. Quarterly Fournal of Speech, 62(4), 361-375. https://doi.org/I0.I080/00335637609383350

De Meulenaer, S., De Pelsmacker, P., \& Dens, N. (20I8). Power distance, uncertainty avoidance, and the effects of source credibility on health risk message compliance. Healtb Communication,33(3), 29I-298. https://doi.org/10.1080/10410236.2016.1266573

Eagly, A. \& Chaiken, S. (1993). The psychology of attitudes. Orlando, FL: Harcourt Brace.

Embacher, K., McGloin, R., \& Richards, K. (20I8). When women give health advice online, do we listen? The effect of source sex on credibility and likelihood to use online health advice. Western Fournal of Communication, 82(4), 439-456. https://doi.org/10.1080/10570314.2017.1367028

Flanagin, A. J. \& Metzger, M. J. (2007). The role of site features, user attributes, and information verification behaviors on the perceived credibility of web-based information. New Media \& Society, 9(2), 319-342. https://doi.org/10.1177/1461444807075015.

Fox, M. (II de julio de 2020). Así afecta el coronavirus todo el cuerpo. CNN Español. Recuperado de: https://cnn.it/3rK2xQN

Gantz,W.(198I).Theinfluenceofresearchermethodsontelevisionandnewspapernewscredibilityevaluations. Fournal of Broadcasting, 25(2), I55-169. https://doi.org/I0.1080/08838158109386439

Gass, R. H. \& Seiter, J. S. (1999). Persuasion, social influence, and compliance gaining. Boston, MA: Allyn \& Bacon. 
Gaziano, C. \& McGrath, K. (I986). Measuring the concept of credibility. Fournalism Quarterly, 63(3), 45I-462. https://doi.org/10.II77/I0776990860630030I

Google (202I). See what was trending in 2020 México. Google. Trends. Recuperado de: https://bit. ly/3 $\mathrm{bxTMU}_{5}$

Greenberg, B. S. \& Roloff, M. E. (1974). Mass media credibility: research results and critical issues. Washington, DC: American Newspaper Publishers Association.

Gunther, A. C. (1988). Attitude extremity and trust in media. Fournalism Quarterly, 65(2), 279-287. https://doi.org/I0.II77/107769908806500203

Gunther, A. C. (1992). Biased press or biased public? Attitudes toward media coverage of social groups. Public Opinion Quarterly, 56(2), I47-I67. https://doi.org/10.1086/269308

Hajli, M. N., Sims, J., Featherman, M. \& Love, P. E. D. (2015). Credibility of information in online communities. Fournal of Strategic Marketing, 23(3), 238-253. https://doi.org/10.1080/096525 $4 \mathrm{X} .2014 .920904$

Hawn, C. (2009). Take two aspirin and tweet me in the morning: how Twitter, Facebook, and other social media are reshaping health care. Health Affairs, 28(2), 36I-368. https://doi.org/I0.I377/ hlthaff.28.2.36I

Hovland, C. I., Janis, I. L., \& Kelley, H. H. (1953). Communication and persuasion. New Haven, CT: Yale University Press.

IAB (marzo, 20I9). Estudio de consumo de medios y dispositivos entre internautas mexicanos. IIa. edición. Ciudad de México, México: Kantar-IAB México-Televisa Digital.

IAB (septiembre, 2020). Estudio de consumo de medios y dispositivos entre internautas mexicanos. I2a. edición. Ciudad de México, México: Kantar-IAB México-Televisa Digital.

IFT (2019). Encuesta nacional de consumo de contenidos audiovisuales 20I9. Ciudad de México, México: Instituto Federal de Telecomunicaciones.

Ihlen, $\emptyset$. (2020). Science communication, strategic communication and rhetoric: the case of health authorities, vaccine hesitancy, trust and credibility. Fournal of Communication Management, 24(3), I63-167. https://doi.org/I0.II08/JCOM-03-2020-00I7

INEGI (202I). Población. Población total. INEGI. Recuperado de: https://bit.ly/3sCNA2n

Jackson, D. N., Peterson, E. B., Blake, K. D., Coa, K. \& Chou, W. Y. S. (2019). Americans' trust in health information sources: trends and sociodemographic predictors. American Fournal of Health Promotion, 33(8), II87-II93. https://doi.org/10.II77/0890117II986I280

Klawitter, E., \& Hargittai, E. (20I8). Shortcuts to well being? Evaluating the credibility of online health information through multiple complementary heuristics. Fournal of Broadcasting \&o Electronic Media, 62(2), 25I-268. https://doi.org/I0.1080/0883815I.20I8.1451863

Kemp, S. (202I). Digital 2020: Mexico. DataReportal. Recuperado de: https://bit.ly/3sFuPLC

Lee, R. S. (1978). Credibility of newspaper and TV news. Fournalism Quarterly, 55(2), 282-287. https://doi.org/I0.II77/107769907805500209

Levinson, P. (2013). New new media. 2a. ed., Nueva York, NY: Penguin Academics. 
Machackova, H., \& Smahel, D. (20I8). The perceived importance of credibility cues for the assessment of the trustworthiness of online information by visitors of health-related websites: The role of individual factors. Telematics \& Informatics, 35(5), I534-I54I. https://doi.org/IO.IoI6/j. tele.2018.03.02I

Masílamani, V., Sriram, A., \& Rozario, A. M. (2020). Alfabetización en e-Salud de los jóvenes: credibilidad y calidad de la información sanitaria con móviles en la India. Comunicar, 28(64), 85-95. https://doi.org/10.3916/C64-2020-08

Meyer, P. (1988). Defining and measuring credibility of newspapers: developing an index. Fournalism Quarterly, 65(3), 567-574. https://doi.org/I0.1177/10776990880650030I

MSP (28 de febrero de 2020). Primer caso de coronavirus en México. Medicina y Salud Pública. Recuperado de: https://bit.ly/2ODy7ki

Newhagen, J. \& Nass, C. (1989). Differential criteria for evaluating credibility of newspapers and TV news. Fournalism Quarterly, 66(2), 277-284. https://doi.org/I0.II77/107769908906600202

O’Keefe, D. J. (I990). Persuasion: theory and research. Newbury Park, CA: Sage.

Palanisamy, B., Gopichandran, V. \& Kosalram, K. (20I8). Social capital, trust in health information, and acceptance of measles-rubella vaccination campaign in Tamil Nadu: a case-control study. Fournal of Postgraduate Medicine, 64(4), 212-219. https://doi.org/10.4103/jpgm.JPGM_249_I7

Petty, R. E., \& Cacioppo, J. T. (198I). Attitudes and persuasion: classic and contemporary approaches. Dubuque, IA: William C. Brown.

Popescu, S. D., Popescu, A. O., Maior, D., Dănilă, M., Dobria, M., \& Nădăşan, V. (2018). Widely accepted credibility criteria for online health-related information are not correlated with content quality of stroke webpages in two languages of Central and Eastern European countries. Fournal of Interdisciplinary Medicine, 3(3), I96-20I. https://doi.org/10.2478/jim-2018-003I

Reagan, J. \& Zenaty, J. (1979). Local news credibility: newspapers vs. TV news revisited. Fournalism Quarterly, 56(I), I68-I72. https://doi.org/I0.II77/I07769907905600I27

Rimmer, T. \& Weaver, D. (1987). Different questions, different answers? Media use and media credibility. Fournalism Quarterly, 64(I), 28-44.https://doi.org/10.1177/107769908706400104

Sbaffi, L. \& Rowley, J. (2017). Trust and credibility in web-based health information: a review and agenda for future research. Fournal of Medical Internet Research, I9(6): I-I6. https://doi. org/10.2196/jmir.7579

Secretaría de Salud (2020). Conferencia 30 de diciembre de 2020. Coronavirus. Recuperado de: https://bit.ly/3v6Vbst

Secretaría de Salud (202I). Información accesible. Coronavirus. Recuperado de: https://bit.ly/315e$\mathrm{Vbh}$

Self, C. C. (1996). Credibility. En M. B. Salwen \& D. W. Stacks (eds.), An integrated approach to communication theory and research (pp. 42I-44I). Mahwah, NJ: Erlbaum.

Shah, Z., Surian, D., Dyda, A., Coiera, E., Mandl, K. D. \& Dunn, A. G. (2019). Automatically appraising the credibility of vaccine-related web pages shared on social media: a Twitter surveillance study. Fournal of Medical Internet Research, 2 I(II), I-I4. https://doi.org/I0.2196/I4007 
Shin, D.-H., Lee, S., \& Hwang, Y. (2017). How do credibility and utility play in the user experience of health informatics services? Computers in Human Bebavior, 67: 292-302. https://doi.org/10.1016/j.chb.2016.11.007

Song, S., Zhang, Y. \& Yu, B. (2020). Interventions to support consumer evaluation of online health information credibility: a scoping review. International Fournal of Medical Informatics, I45, I-II. https://doi.org/I0.IoI6/j.ijmedinf.2020.10432I

Stamm, K. \& Dube, R. (1994). The relationship of attitudinal components to trust in media. Communication Research, 2I(I), 105-123. https://doi.org/I0.1177/009365094021001006

Thon, F. M., \& Jucks, R. (2017). Believing in expertise: how authors' credentials and language use influence the credibility of online health information. Health Communication, 32(7), 828-836. https://doi.org/I0.1080/10410236.2016.II72296

Van der Weerd, W., Timmermans, D. R., Beaujean, D.J., Oudhoff,J. \& Van Steenbergen,J.E. (20II). Monitoring the level of government trust, risk perception and intention of the general public to adopt protective measures during the influenza $\mathrm{A}(\mathrm{HINI})$ pandemic in the Netherlands. $B M C$ Public Health, II, 575. https://doi.org/IO.II86/I47I-2458-II-575

Valizadeh-Haghi, S., Rahmatizadeh, S., Ansari, M., \& Hamzehei, R. (20I8). Credibility of health websites on infectious diseases: Are there any fully trustable website to read on Ebola? Archives of Advances in Biosciences, 9(3), 23-31. https://doi.org/I0.22037/jps.v9i3.19330

Vidgen, R., Sims, J. \& Powell, P. (2013). Do CEO bloggers build community? Fournal of Communication Management, I7(4),364-385. https://doi.org/I0.IIO8/JCOM-08-20I2-0068

Xiang, P., Guo, J., Liu, Q. (2020). How government credibility and social morality work in a public health emergency. A study of public quarantine willingness in COVID-19. Analyses of Social Issues or Public Policy, 20(I): 443-46r. https://doi.org/Io.III/asap.I2209

Esta obra está bajo Licencia Creative Commons Atribución-NoComercial-SinDerivadas 4.o Internacional. 